\title{
Besoins en matière de soins de soutien des patients ayant un cancer avancé et subissant une radiothérapie pour en atténuer les symptômes
}

\author{
par Margaret I. Fitch
}

\section{Abrégé}

Beaucoup de patients atteints d'un cancer avancé ont de nombreu ses complications médicales et de multiples sites où les métastases causent des symptômes pénibles. On utilise souvent la radiothérapie à des fins palliatives chez ces patients, particulièrement chez ceux ayant des métastases osseuses. Pourtant, on possède peu d'information sur les types de besoins que ces patients éprouvent en matière de soins de soutien, sur les services qui leur sont offerts et si les patients veulent de l'aide pour ces besoins.

Le but principal de cette étude descriptive transversale était d'identifier les besoins en matière de soins de soutien (physiques, affectifs, sociaux, spirituels, psychologiques et pratiques) des patients atteints d'un cancer avancé qui fréquentaient la Clinique d'intervention rapide en radiothérapie palliative (CIRRP) d'un centre intégré de cancérologie ambulatoire. Son second but était de déterminer si les patients voulaient recevoir de l'aide afin de répondre à ces besoins.

Soixante-neuf patients ont participé à cette étude en remplissant un questionnaire d'autoévaluation. Les données fournissaient une nette indication qu'une gamme de besoins en matière de soins de soutien demeurait non satisfaite parmi ce groupe de patients. Le manque d'énergie, la douleur et les inquiétudes relatives au tracas que se faisait l'entourage étaient les besoins les plus fréquemment signalés. De plus, les patients exprimaient divers degrés de difficulté sur le plan de la gestion des besoins, et beaucoup d'entre eux souhaitaient qu'on les aide à gérer les besoins dégagés. Cependant, malgré ce qui précède, un nombre important des patients indiquaient qu'ils ne souhaitaient pas recevoir d'assistance vis-à-vis de certains besoins. Des suggestions pour la pratique et la recherche à venir sont offertes afin d'aider les infirmières en oncologie à dispenser des soins de soutien à ces patients.

Beaucoup de patients atteints d'un cancer avancé ont de nombreuses complications médicales et de multiples sites où les métastases causent des symptômes pénibles (Andersson \& Sousa, 1998). On utilise souvent la radiothérapie à des fins palliatives chez ces patients (Ciezki, Komurcu \& Macklis, 2000; Kirkbride, 1995). Les patients ayant des métastases osseuses éprouvent de la douleur et subissent une limitation de mobilité (Body, 1992) lesquelles peuvent être soulagées au moyen d'une seule radiothérapie. Cependant, beaucoup de patients nécessitent une brève série de traitements (c.-à-d. plusieurs fractions) afin de soulager la douleur. La Clinique d'intervention rapide en radiothérapie palliative (CIRRP) a été instituée dans un centre intégré de cancérologie ambulatoire en vue de fournir un accès rapide à la radiothérapie à court terme pour les patients présentant des symptômes associés au cancer avancé et d'améliorer ainsi leur qualité de vie (Andersson \& Sousa, 1998). Ces patients se rendent au centre de cancérologie pour leur traitement de

\section{Au sujet d'auteure}

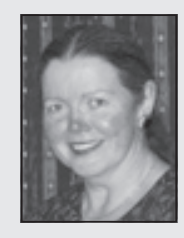

Margaret I. Fitch, inf., Ph.D., Chef, Soins infirmiers en oncologie, co-directrice, Soutien au patient et à la famille, Centre de cancérologie Odette au Sunnybrook, 2075 Bayview Avenue, T-wing, Toronto, ON M4N 3M5. Téléphone: 416-480-5891; Téléc.: 416-480-7806; Courriel: marg.fitch@sunnybrook.ca radiothérapie, parfois administré en une seule fois, avant de retourner chez eux ou à l'hôpital pour y recevoir les soins de suivi. La plupart des patients sont évalués par le ou la radio-oncologue, l'infirmière en oncologie et le ou la technicien(ne) en radiothérapie; passent par l'étape de planification et de simulation; et enfin, reçoivent leur traitement de radiothérapie et ce, durant la même journée.

Les professionnels de la santé dispensant des soins aux patients atteints d'un cancer avancé de notre centre de soins ambulatoires, plus spécialement le personnel infirmier, avaient exprimé des doutes quant à savoir si le centre de cancérologie fournissait des soins à un niveau répondant aux besoins de cette population. De plus, ils ont souligné le manque d'information sur les types de besoins en matière de soins de soutien éprouvés par ces patients, sur les services qui sont disponibles, si les patients veulent qu'on les aide relativement à leurs besoins, et qui aborde ces besoins, le cas échéant. En réponse à ces doutes, on a entrepris une exploration systématique des besoins en matière de soins de soutien des patients atteints d'un cancer avancé. Notre but ultime était d'optimaliser la qualité des soins dispensés à cette clientèle du centre de cancérologie. La CIRRP a été sélectionnée comme premier site où réaliser l'enquête puisque tous ses patients appartiennent à la catégorie des porteurs de cancers avancés.

\section{Analyse documentaire}

Le cancer et son traitement ont des répercussions physiques, sociales, affectives, psychologiques, spirituelles et pratiques pour les personnes atteintes de cancer (Ashbury, Findley, Reynolds \& McKerracher, 1998; Charles, Sellick, Montesanto \& Mohide, 1996; Fitch, Porter \& Page, 2008; Whelan et al., 1997). À mesure que la maladie évolue et que les symptômes deviennent plus prononcés, les patients et les membres de la famille peuvent avoir du mal à gérer leurs activités quotidiennes et à composer avec la situation. Bon nombre de patients ayant un cancer avancé sont polysymptomatiques. Des symptômes physiques comme la fatigue (Bradley, Davis \& Chow, 2005), la douleur (Bradley et al., 2005; Bruera et al., 2001), l'anorexie (Hutton et al., 2006; Sarna, Lindsey, Dean, Brecht \& McCorkle, 1994), la toux (Sarna et al., 2004), un faible sentiment de bien-être (Bradley et al.; Bruera et al., 2001) et l'essoufflement (Bradley et al., 2005; Edmonds, Karlsen, Khan \& Addington-Hall, 2001) sont fréquents et ont une incidence négative sur la vie des patients atteints de cancer avancé et de leur entourage. Dans un exposé de synthèse, Solano et Higginson (2006) analysaient 33 articles signalant la prévalence de 11 symptômes chez les patients ayant un cancer avancé (la taille des échantillons variait de 2888 à 10379 patients pour chacun des symptômes). Quoiqu'il existe une gamme étendue sur le plan de la prévalence rapportée parmi les études, la douleur, la fatigue et l'essoufflement étaient généralement présents chez plus de $50 \%$ des patients ayant un cancer avancé.

D’autres symptômes comme la dépression et l'anxiété (Bradley et al., 2005; Bruera et al., 2001) sont eux aussi fréquemment présents et ont un effet adverse sur la qualité de vie des patients. Bradley et ses collègues indiquaient que $61 \%$ des patients fréquentant leur clinique souffraient de dépression $(\mathrm{n}=1070)$. La dépression allait de modérée à grave chez $30 \%$ de ces patients. De plus, $78 \%$ d'entre eux faisaient état d'anxiété $(\mathrm{n}=1079)$, et que cette dernière atteignait des niveaux graves chez 121 patients (11\%). Solano et Higginson (2006) remarquaient également de hauts degrés d'anxiété et de dépression dans leur synthèse sur les patients ayant un cancer avancé (taille des échantillons: 3274 et 4378 patients, respectivement). 
Bien que nous possédions des informations sur les besoins en matière de soins de soutien des patients ayant un cancer avancé, il existe peu de connaissances sur l'éventail complet des besoins des patients externes en phase palliative, sur les besoins qui demeurent insatisfaits et sur le fait de savoir si les patients veulent de l'aide ou non au niveau de ces besoins non satisfaits. Une telle information est importante pour plusieurs raisons: 1) planifier les soins individuels du patient, 2) prendre des décisions en matière d'affectation des ressources, et, 3) cerner les domaines où la qualité de la prestation de soins pourrait être améliorée.

\section{But}

Le but principal de cette étude descriptive transversale était d'identifier les besoins en matière de soins de soutien (physiques, affectifs, sociaux, spirituels, psychologiques et pratiques) des patients atteints d'un cancer avancé qui fréquentaient la Clinique d'intervention rapide en radiothérapie palliative (CIRRP) d'un centre intégré de cancérologie ambulatoire. Son but secondaire était de déterminer si les patients voulaient recevoir de l'aide afin de répondre à ces besoins.

\section{Méthodologie}

\section{Procédures-Recrutement et collecte de données}

L'étude a débuté après qu'on a reçu l'approbation du Comité d'éthique de la recherche de Sunnybrook. Une assistante de recherche a consulté le personnel de la CIRRP en vue d'identifier les participants éventuels. Tous les patients fréquentant la CIRRP pouvaient être inclus dans l'étude à moins de vivre une détresse grave et d'être incapables de se concentrer sur le remplissage du questionnaire. Cette étude comprenait un échantillon de commodité de patients ayant une gamme de diagnostics de cancer et ayant tous reçu un diagnostic médical de cancer avancé. L'assistante de recherche abordait les patients admissibles dans la zone d'accueil de la clinique et leur expliquait les objectifs et méthodes de l'étude. Les patients ont tous été mis au courant, verbalement, de leurs droits quant à la participation à l'étude, et l'assistante de recherche les a ensuite invités à participer. Ceux qui ont accepté de le faire ont signé un formulaire de consentement en double. Les patients en gardaient une copie tandis que l'assistante de recherche en conservait la seconde.

L'assistante de recherche a remis à chaque participant un questionnaire d'autoévaluation à remplir et a évalué si le patient avait besoin d'aide pour ce faire. S'il avait besoin d'aide à cause d'une déficience physique, l'assistante de recherche l'emmenait dans un local privé et remplissait le questionnaire dans le cadre d'une entrevue. En ce qui concerne les patients qui avaient accepté de remplir eux-mêmes le questionnaire lors de leur visite à la clinique, l'assistante de recherche revenait voir chacun d'eux à un moment prédéterminé afin de recueillir le questionnaire dûment rempli. Il y avait également une boîte à la clinique dans laquelle les patients pouvaient laisser leurs questionnaires après avoir mis ceux-ci dans des enveloppes et scellé ces dernières. Le questionnaire se remplissait en 15-20 minutes. Finalement, si un patient n'avait pas la possibilité de remplir le questionnaire durant sa visite à la clinique, l'assistante de recherche lui fournissait une enveloppe affranchie et pré-adressée dans laquelle il pouvait renvoyer le questionnaire. La soumission des questionnaires remplis était laissée au gré des participants, et aucun rappel ne leur a été envoyé.

L'outil de mesure (dont la description est fournie ci-dessous) n'était disponible qu'en anglais. Afin de minimiser l'exclusion de patients qui ne parlaient ni ne lisaient l'anglais et/ou n'écrivaient pas dans cette langue, on invitait les membres de la famille ou amis qui parlaient couramment l'anglais à expliquer l'étude à ces patients et à leur fournir une traduction du contenu si le proche ou l'ami et le patient étaient d'accord. Le patient signait alors le formulaire de consentement, ainsi que l'interprète qui y signalait par écrit que le patient avait librement donné son consentement. L'assistante de recherche notait la manière dont les questionnaires étaient remplis (directement par le patient, dans le cadre d'une entrevue avec l'assistante de recherche ou par l'intermédiaire d'un(e) interprète). L'analyse n'a révélé aucune différence entre ces groupes pour ce qui est des taux de réponse. Quand les patients donnaient leur permission dans le formulaire de consentement, l'assistante de recherche accédait à leurs dossiers afin de remplir la fiche de renseignements démographiques et d'inscrire la date où les métastases avaient été diagnostiquées pour la première fois chez les patients.

\section{Instrument de collecte des données}

Le questionnaire utilisé dans le cadre de cette étude se composait de deux parties. La composante démographique a été élaborée aux fins de la présente étude tandis que l'évaluation des besoins en matière de soins de soutien était une adaptation d'un outil tiré de Bonevski et al. (2000).

La fiche de données démographiques comprenait huit questions : âge en années, sexe; situation maritale; plus haut niveau de scolarité atteint; type de cancer; mois et année où le cancer a été diagnostiqué pour la première fois; traitement reçu pour le cancer; et traitement anticancéreux actuellement dispensé. La Supportive Care Needs Survey [Enquête sur les besoins en matière de soins de soutien] était à la base du questionnaire utilisé dans la présente étude. La Supportive Care Needs Survey avait été initialement élaborée et validée en Australie (Bonevski et al., 2000). Une analyse factorielle en composantes principales révélait que les items relatifs aux besoins pouvaient être regroupés selon les cinq facteurs suivants: besoins physiques et vie quotidienne, besoins psychologiques, système de santé et information, soins et soutien du patient, et enfin, sexualité. Les coefficients alpha de Cronbach correspondant à ces facteurs ou sous-échelles s'étendaient de 0,87 à 0,97 . L'outil original se composait de 60 questions fermées. Les patients y répondaient au moyen d'une échelle de type Likert en cinq points allant de 0 à 4 où « 0 » signifiait «Aucun besoin» d'aide (l'item n'était pas un enjeu pour le patient du fait de son cancer) et « 4 » signifiait un «grand besoin» d'aide (l'item constituait une inquiétude majeure ou revêtait une grande importance pour le patient, et celui-ci avait grand besoin d'une aide supplémentaire au niveau de ce problème ou de cette inquiétude). L'outil peut être compris par les personnes peu scolarisées et rempli en l'espace de 20 minutes, environ (Bonevski et al., 2000).

La présente étude a utilisé la version révisée Supportive Care Needs Survey-Radiation (Enquête sur les besoins en matière de soins de soutien-Radiothérapie) de la Supportive Care Needs Survey originale (Bonevski et al., 2000). Un essai pilote de l'outil original de Bonevski avait été réalisé auprès de patients ayant le cancer du poumon dans notre centre de cancérologie situé au Canada et avait débouché sur le réordonnancement de quelques questions dans un souci de clarté et sur de menus changements de mots afin de refléter le contexte ambulatoire canadien. Par exemple, le terme «hôpital» a été remplacé par «centre de cancérologie». De plus, la version révisée de l'outil incluait une question supplémentaire après chaque item qui demandait au patient s'il souhaitait recevoir de l'aide relativement à ce besoin particulier. Ainsi, pour chaque item, on demandait aux patients d'indiquer s'ils souhaitaient recevoir de l'aide concernant le besoin en question (Non, je ne veux aucune aide; Oui, je voudrais de l'aide; Je suis incertain(e) de vouloir de l'aide). La version révisée de l'enquête comportait 61 items répartis entre sept domaines de soins de soutien (voir le tableau 1). Les scores de fidélité pour le groupe de patients atteints d'un cancer du poumon étaient compris entre 0,66 et 0,90, six des sept sous-échelles de domaine ayant des scores égaux ou supérieurs à 0,80 (Fitch \& Steele, 2010b). Dans la présente étude, les coefficients alpha de Cronbach correspondant aux sept sous-échelles de domaines variaient de 0,35 (besoins pratiques) à 0,81 (besoins d'information) (voir le tableau 1). Il n'existait pas de différences significatives dans les sous-échelles de besoins en matière de soutien à l'exception de la sous-échelle des 
besoins pratiques où les femmes avaient des résultats moyens légèrement plus élevés que ceux des hommes $(t=2,0157, p=0,0478)$. Par conséquent, les résultats sont présentés pour l'ensemble du groupe de participants.

\section{Analyse des données}

L'analyse des données sur les besoins en matière de soins de soutien était descriptive et se concentrait sur le calcul de la fréquence des items et des scores moyens et des écarts-types des sous-échelles. Des tests de différence ont été utilisés afin de déterminer l'influence de variables démographiques choisies sur le degré du besoin exprimé (tests-t pour le sexe et le niveau de scolarité; ANOVA pour les variables nominales situation maritale, temps écoulé depuis le diagnostic et âge). Le seuil de signification a été fixé à $\mathrm{p}<0,05$ pour tous les tests, et le logiciel de statistique SPSS a été utilisé dans le cadre de l'analyse.

\section{Résultats}

\section{Échantillon}

Cent six $(n=106)$ patients ont été abordés dans la salle d'attente sur une période de neuf mois et 95 d'entre eux ont accepté de participer à l'étude. Onze patients ont verbalement décliné l'invitation quand ils ont été contactés par l'assistante de recherche parce qu'ils ne voulaient pas participer à une étude de recherche, ne se sentant pas d'attaque ou ils ont indiqué qu'ils seraient peut-être intéressés à un moment ultérieur. Deux autres patients n’ont pas donné de raison à l'assistante de recherche. Sur les 93 questionnaires remis aux patients, quelques-uns n'ont pas été renvoyés par la poste; on s'est aperçu, à la suite d'un examen, que trois personnes n'étaient pas admissibles (n'étant pas des patients de la clinique du Programme d'intervention rapide en radiothérapie), et il manquait, dans plusieurs questionnaires, des données pour quatre ou plus items, et ceux-ci étaient jugés inutilisables. La taille finale de l'échantillon se montait à 69 patients.

Il y avait un nombre presque égal d'hommes et de femmes dans l'échantillon (voir le tableau 2). Semblable en cela à la clientèle de la clinique, l'échantillon était d'âge mûr avec un âge moyen de 65 ans, la plupart des patients étant mariés $(n=52)$, et presque un quart d'entre eux ayant au moins une scolarité de niveau postsecondaire. Environ 45\% d'entre eux avaient été diagnostiqués moins d'un an auparavant. Les patients étaient atteints de divers types de cancers, comme le cancer du sein, les cancers gastro-intestinaux, le cancer de la prostate et le cancer du poumon. Approximativement $75 \%$ d'entre eux ont signalé suivre actuellement un traitement. Tous indiquaient avoir reçu, auparavant, de la chimiothérapie, de la radiothérapie, de la chirurgie ou une combinaison de ces traitements.

Enjeux actuellement éprouvés les plus fréquemment rapportés

Les patients ont signalé qu'ils éprouvaient actuellement des besoins en matière de soins de soutien dans les sept domaines

\section{Tableau 1. Supportive Care Needs Survey (SCNS) (61 items)} [Enquête sur les besoins en matière de soins de soutien]

\begin{tabular}{|l|c|c|}
\hline Domaine & Nombre d'items & $\begin{array}{c}\text { Coefficients alpha } \\
\text { de Cronbach }\end{array}$ \\
\hline B. d'information & 9 & 0,81 \\
\hline Symptômes physiques & 11 & 0,78 \\
\hline B. psychologiques & 11 & 0,74 \\
\hline B. affectifs & 11 & 0,72 \\
\hline B. spirituels & 7 & 0,71 \\
\hline B. sociaux & 5 & 0,43 \\
\hline B. pratiques & 7 & 0,35 \\
\hline
\end{tabular}

et pour presque tous les items. Le nombre de patients éprouvant un enjeu particulier allait de 0 (p. ex. besoins physiques: «que le personnel de l'hôpital s'occupe promptement de vos besoins physiques »; besoins sociaux: "que vos proches et amis aient la permission d'être avec vous à l'hôpital quand vous le désirez») à 53 (p. ex. besoins physiques: «le manque d'énergie»). Le domaine de l'information était le seul domaine où il n'y avait pas au moins un item où un quart ou plus des patients éprouvaient actuellement un problème dans le domaine. Les tableaux 3 à 9 présentent les données relatives à chacun des domaines.

Onze enjeux ont été signalés comme étant actuellement éprouvés par plus de $50 \%$ des patients. Six enjeux se rapportaient au domaine physique (c.-à-d. le manque d'énergie, la douleur, ne pas être capable de faire des choses que vous faisiez auparavant, ne pas être capable de faire les tâches ménagères, se sentir mal, et enfin, perte d'appétit); trois relevaient du domaine psychologique (c.-à-d. craintes relatives à la déficience ou à la détérioration physique, crainte que le cancer ne se propage, et enfin, craintes relatives à la douleur); un était de nature sociale (c.-à- d. s'inquiéter du souci que se font les personnes qui vous sont chères); et un, relevait du domaine affectif (c.-à-d. se sentir abattu ou déprimé) (voir le tableau 10).

\section{Désir d'être aidé(e)}

On demandait aux patients qui indiquaient éprouver actuellement un enjeu particulier de signaler également s'ils souhaitaient recevoir de l'aide afin de composer avec ce besoin. Nous avons posé l'hypothèse que toute personne qui était «incertaine» de vouloir de l'aide ou pas méritait une conversation avec le personnel. Nous avons donc combiné le nombre de patients qui choisissait «incertain(e)» de vouloir de l'aide avec le nombre de patients qui répondaient «oui». Ce total a été divisé par le nombre de participants qui signalaient éprouver actuellement l'enjeu. Ces calculs déterminaient les pourcentages de patients éprouvant actuellement un enjeu qui désiraient un type quelconque d'aide. Les résultats se rapportant aux patients qui ont choisi soit «oui» soit «incertain(e)» sont présentés dans les tableaux 3 à 9. L'étendue des pourcentages de patients faisant face à un enjeu qui désiraient possiblement recevoir de l'aide à son sujet variait d'un domaine à l'autre: b. psychologiques, de 28,6 à 100\%; b. pratiques de 28 à 100\%; b. affectifs, de 23,5 à 100\%; b. d'information, de 50 à $85,7 \%$; b. physiques, de 33,3 à $63,5 \%$; b. spirituels, de 25 à 55,6\%; et enfin, b. sociaux, de 29 à $45,5 \%$. Toutefois, il convient de remarquer qu'il s'agit d'un petit échantillon et que le nombre absolu de personnes voulant de l'aide est peu élevé (voir le tableau 10).

\begin{tabular}{|c|c|}
\hline Variable & Résultat (n, \%) \\
\hline Sexe & $\begin{array}{l}\text { Masculin=35 }(50,7 \%) \\
\text { Féminin = } 34(49,3 \%)\end{array}$ \\
\hline Âge & $\begin{array}{l}\text { Moyenne }=65 \text { ans } \\
\text { Étendue }=35-84 \text { ans }\end{array}$ \\
\hline Situation maritale & $\begin{array}{l}\text { Marié(e) }=52(75,4 \%) \\
\text { Séparé(e)/divorcé(e)/veuf(ve)=15 (21,7\%) } \\
\text { Célibataire (jamais marié(e))=2 (2,9\%) }\end{array}$ \\
\hline Niveau de scolarité & $\begin{array}{l}<\text { Études postsecondaires }=52(75,4 \%) \\
\geq \text { Études postsecondaires }=17(24,6 \%)\end{array}$ \\
\hline $\begin{array}{l}\text { Temps écoulé depuis } \\
\text { le diagnostic }\end{array}$ & $\begin{array}{l}<1 \text { an }=31(45,6 \%) \\
1-2 \text { ans }=9(13,2 \%) \\
2+\text { ans }=28(41,2 \%)\end{array}$ \\
\hline \multicolumn{2}{|c|}{$\begin{array}{l}\text { * Comme des données manquent dans certains cas, les totaux ne } \\
\text { sont pas toujours égaux à } 69 .\end{array}$} \\
\hline
\end{tabular}




\section{Discussion}

Cette étude a été menée en vue d'explorer les besoins en matière de soins de soutien des patients ayant un cancer avancé et fréquentant une clinique de radiothérapie pour le soulagement de leurs symptômes. Elle fournit un instantané de l'étendue des besoins physiques et psychosociaux éprouvés par ces patients ainsi qu'une indication de la mesure dans laquelle leurs besoins demeuraient insatisfaits et s'ils voulaient recevoir de l'aide relativement à ces derniers.
Les symptômes précédemment dégagés dans la littérature étaient également présents chez les patients ayant participé à cette étude. Malgré la vaste étendue de la prévalence des symptômes entre les diverses études, la douleur, la fatigue et l'essoufflement étaient généralement présents chez plus de $50 \%$ des patients ayant un cancer avancé (Solano \& Higginson, 2006). Dans la présente étude, la fatigue (le manque d'énergie) $(76,8 \%)$ et la douleur $(75,4 \%)$ étaient les enjeux éprouvés les plus souvent rapportés par les patients de la CIRRP, et 32 des 69 (46,4\%) patients signalaient l'essoufflement.

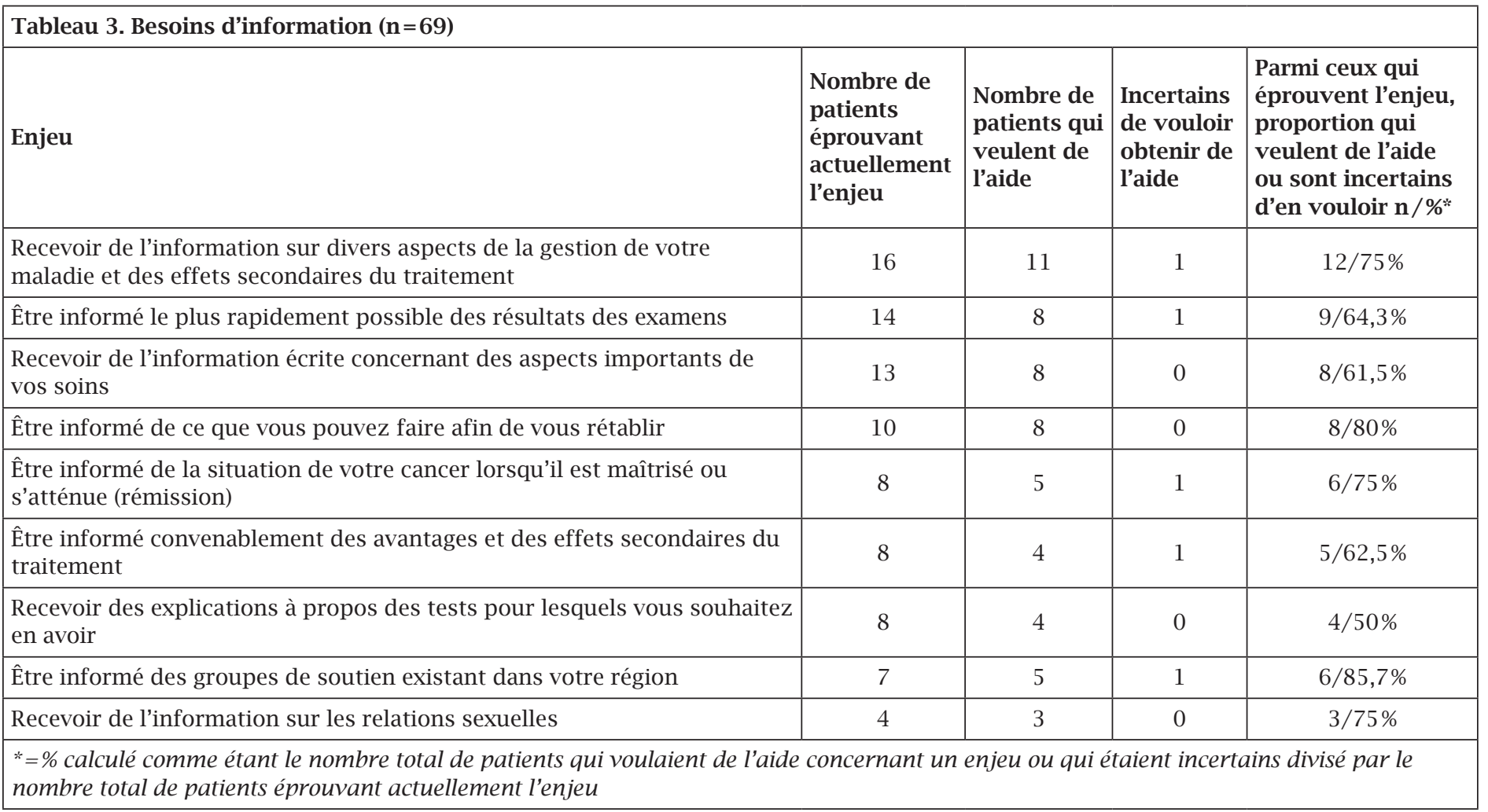

Tableau 4. Besoins physiques $(n=69)$

\begin{tabular}{|c|c|c|c|c|}
\hline Enjeu & $\begin{array}{l}\text { Nombre de } \\
\text { patients } \\
\text { éprouvant } \\
\text { actuellement } \\
\text { l'enjeu }\end{array}$ & $\begin{array}{l}\text { Nombre de } \\
\text { patients qui } \\
\text { veulent de } \\
\text { l'aide }\end{array}$ & $\begin{array}{l}\text { Incertains } \\
\text { de vouloir } \\
\text { obtenir de } \\
\text { l'aide }\end{array}$ & $\begin{array}{l}\text { Parmi ceux qui éprouvent } \\
\text { l'enjeu, proportion qui } \\
\text { veulent de l'aide ou sont } \\
\text { incertains d'en vouloir } \\
\text { n/\%* }\end{array}$ \\
\hline Manque d'énergie & 53 & 24 & 4 & $28 / 52,8 \%$ \\
\hline Douleur & 52 & 31 & 2 & $33 / 63,5 \%$ \\
\hline Ne pas être capable de faire ce que vous pouviez faire auparavant & 49 & 20 & 3 & $23 / 46,9 \%$ \\
\hline Ne pas être capable de faire les travaux ménagers & 43 & 17 & 2 & $19 / 44,2 \%$ \\
\hline Ne pas se sentir bien & 38 & 13 & 4 & $17 / 44,7 \%$ \\
\hline Perte d'appétit & 38 & 15 & 3 & $18 / 47,4 \%$ \\
\hline Essoufflement & 32 & 12 & 2 & $14 / 43,8 \%$ \\
\hline Toux & 21 & 6 & 4 & $10 / 47,6 \%$ \\
\hline Nausée/vomissements & 20 & 8 & 0 & $8 / 40 \%$ \\
\hline Changements au niveau de la libido & 12 & 4 & 0 & $4 / 33,3 \%$ \\
\hline $\begin{array}{l}\text { Que le personnel hospitalier s'occupe promptement de vos } \\
\text { besoins physiques }\end{array}$ & 0 & 0 & 0 & S.O. \\
\hline
\end{tabular}


Les autres symptômes précédemment dégagés dans la littérature et qui étaient signalés par plus de $50 \%$ des patients de notre étude incluaient l'anorexie (Hutton et al., 2006), un faible sentiment de bien-être (Bruera et al., 2001; Bradley et al., 2005) et la dépression (Bruera et al., 2001, Bradley et al., 2005, Solano \& Higginson, 2006). Quoiqu'ils ne figurent pas parmi les principaux enjeux rapportés dans la présente étude, des symptômes courants dont il a été fait état dans la littérature existaient également dans notre échantillon, notamment la toux (Sarna et al., 2004) et l'anxiété (Bruera et al., 2001; Bradley et al., 2005; Solano \& Higginson, 2006),

Il est intéressant de noter que ce n'est pas parce qu'un grand nombre de patients éprouvaient actuellement un enjeu qu'ils suite à la page 98.

\begin{tabular}{|c|c|c|c|c|}
\hline \multicolumn{5}{|l|}{ Tableau 5. Besoins affectifs $(n=69)$} \\
\hline Enjeu & $\begin{array}{l}\text { Nombre de } \\
\text { patients } \\
\text { éprouvant } \\
\text { actuellement } \\
\text { l'enjeu }\end{array}$ & $\begin{array}{l}\text { Nombre de } \\
\text { patients } \\
\text { qui veulent } \\
\text { de l'aide }\end{array}$ & $\begin{array}{l}\text { Incertains } \\
\text { de vouloir } \\
\text { obtenir de } \\
\text { l'aide }\end{array}$ & $\begin{array}{l}\text { Parmi ceux qui } \\
\text { éprouvent l'enjeu, } \\
\text { proportion qui } \\
\text { veulent de l'aide } \\
\text { ou sont incertains } \\
\text { d'en vouloir } \mathrm{n} / \%^{*}\end{array}$ \\
\hline Se sentir abattu(e) ou déprimé(e) & 40 & 10 & 2 & $12 / 30 \%$ \\
\hline Sentiments de tristesse & 34 & 4 & 4 & $8 / 23,5 \%$ \\
\hline Sentiments d'ennui et/ou d'inutilité & 34 & 7 & 4 & $11 / 32,4 \%$ \\
\hline Anxiété & 34 & 6 & 6 & $12 / 35,3 \%$ \\
\hline S'inquiéter que les résultats du traitement échappent à votre contrôle & 25 & 9 & 3 & $12 / 48 \%$ \\
\hline Anxiété à l'idée de recevoir un quelconque traitement & 24 & 11 & 1 & $12 / 50 \%$ \\
\hline Changements au niveau des relations sexuelles & 16 & 3 & 4 & $7 / 43,8 \%$ \\
\hline $\begin{array}{l}\text { La possibilité de parler avec quelqu'un qui comprend et qui a traversé une } \\
\text { expérience similaire }\end{array}$ & 8 & 5 & 0 & $5 / 62,5 \%$ \\
\hline Avoir un membre du personnel hospitalier à qui parler & 6 & 2 & 2 & $4 / 66,7 \%$ \\
\hline Être traité comme une personne et non comme un numéro & 6 & 2 & 1 & $3 / 50 \%$ \\
\hline Que le personnel hospitalier reconnaisse vos sentiments et y soit sensible & 2 & 1 & 1 & $2 / 100 \%$ \\
\hline
\end{tabular}

\begin{tabular}{|c|c|c|c|c|}
\hline \multicolumn{5}{|l|}{ Tableau 6. Besoins psychologiques $(n=69)$} \\
\hline Enjeu & $\begin{array}{l}\text { Nombre de } \\
\text { patients } \\
\text { éprouvant } \\
\text { actuellement } \\
\text { l'enjeu }\end{array}$ & $\begin{array}{l}\text { Nombre de } \\
\text { patients } \\
\text { qui } \\
\text { veulent de } \\
\text { l'aide }\end{array}$ & \begin{tabular}{|l|} 
Incertains \\
de \\
vouloir \\
obtenir \\
de l'aide
\end{tabular} & \begin{tabular}{|l|} 
Parmi ceux qui \\
éprouvent l'enjeu, \\
proportion qui \\
veulent de l'aide \\
ou sont incertains \\
d'en vouloir $\mathbf{n} / \%^{*}$
\end{tabular} \\
\hline Craintes relatives à la déficience ou à la détérioration physique & 42 & 17 & 4 & $21 / 50 \%$ \\
\hline Crainte que le cancer ne se propage & 35 & 15 & 3 & $18 / 51,4 \%$ \\
\hline Craintes relatives à la douleur & 35 & 19 & 3 & $22 / 62,9 \%$ \\
\hline Craintes relatives à la perte d'autonomie & 29 & 11 & 2 & $13 / 44,8 \%$ \\
\hline Accepter les changements sur le plan de l'apparence physique & 17 & 7 & 1 & $8 / 47,1 \%$ \\
\hline Crainte que le cancer ne revienne & 14 & 7 & 3 & $10 / 71,4 \%$ \\
\hline Apprendre à sentir que vous maîtrisez la situation & 14 & 3 & 1 & $4 / 28,6 \%$ \\
\hline $\begin{array}{l}\text { Avoir accès à des consultations professionnelles si vous ou vos proches } \\
\text { en avez besoin }\end{array}$ & 5 & 4 & 0 & $4 / 80 \%$ \\
\hline Être rassuré(e) par le personnel médical que ce que vous ressentez est normal & 4 & 2 & 1 & $3 / 75 \%$ \\
\hline Être traité(e) dans un hôpital ou une clinique où l'ambiance est plaisante & 3 & 1 & 0 & $1 / 33,3 \%$ \\
\hline $\begin{array}{l}\text { Que vos droits au respect de votre vie privée soient mieux protégés } \\
\text { lorsque vous êtes à l’hôpital }\end{array}$ & 2 & 1 & 1 & $2 / 100 \%$ \\
\hline
\end{tabular}


Tableau 7. Besoins spirituels $(n=69)$

\begin{tabular}{|c|c|c|c|c|}
\hline Enjeu & $\begin{array}{l}\text { Nombre de } \\
\text { patients } \\
\text { éprouvant } \\
\text { actuellement } \\
\text { l'enjeu }\end{array}$ & $\begin{array}{l}\text { Nombre de } \\
\text { patients } \\
\text { qui veulent } \\
\text { de l'aide }\end{array}$ & $\begin{array}{l}\text { Incertains } \\
\text { de vouloir } \\
\text { obtenir de } \\
\text { l'aide }\end{array}$ & $\begin{array}{l}\text { Parmi ceux qui éprouvent } \\
\text { l'enjeu, proportion qui } \\
\text { veulent de l'aide ou sont } \\
\text { incertains d'en vouloir } \\
\text { n/\%* }\end{array}$ \\
\hline Incertitude au sujet de l'avenir & 29 & 9 & 4 & $13 / 44,8 \%$ \\
\hline Émotions relatives à la mort et au mourir & 22 & 6 & 1 & $7 / 31,8 \%$ \\
\hline Confusion quant aux raisons pour lesquelles ceci vous est arrivé & 22 & 9 & 0 & $9 / 40,9 \%$ \\
\hline Donner un sens à cette expérience & 13 & 3 & 1 & $4 / 30,8 \%$ \\
\hline Tirer le meilleur parti de votre temps & 12 & 3 & 0 & $3 / 25 \%$ \\
\hline Rester positif(ve) & 9 & 5 & 0 & $5 / 55,6 \%$ \\
\hline Que le personnel hospitalier soit source d'espoir & 2 & 1 & 0 & $1 / 50 \%$ \\
\hline
\end{tabular}

Tableau 8. Besoins sociaux $(n=69)$

\begin{tabular}{|c|c|c|c|c|}
\hline Enjeu & $\begin{array}{l}\text { Nombre de } \\
\text { patients } \\
\text { éprouvant } \\
\text { actuellement } \\
\text { l'enjeu }\end{array}$ & $\begin{array}{l}\text { Nombre de } \\
\text { patients } \\
\text { qui veulent } \\
\text { de l'aide }\end{array}$ & $\begin{array}{l}\text { Incertains } \\
\text { de vouloir } \\
\text { obtenir de } \\
\text { l'aide }\end{array}$ & $\begin{array}{l}\text { Parmi ceux } \\
\text { qui éprouvent } \\
\text { l'enjeu, } \\
\text { proportion qui } \\
\text { veulent de l'aide } \\
\text { ou sont incertains } \\
\text { d'en vouloir } n / \% \text { " }\end{array}$ \\
\hline S'inquiéter du souci que se font les personnes qui vous sont chères & 51 & 12 & 3 & $15 / 29,40 \%$ \\
\hline S’inquiéter de la capacité de vos proches à composer avec votre soin & 31 & 7 & 2 & $9 / 29 \%$ \\
\hline Parler du cancer avec autrui & 11 & 4 & 1 & $5 / 45,5 \%$ \\
\hline Changements relatifs aux attitudes et comportements d'autrui envers vous & 9 & 3 & 0 & $3 / 33,30 \%$ \\
\hline $\begin{array}{l}\text { Que vos proches et amis puissent être avec vous à l'hôpital chaque fois que } \\
\text { vous le désirez }\end{array}$ & 0 & 0 & 0 & S.O. \\
\hline
\end{tabular}

Tableau 9. Besoins pratiques $(n=69)$

\begin{tabular}{|c|c|c|c|c|}
\hline Enjeu & $\begin{array}{l}\text { Nombre de } \\
\text { patients } \\
\text { éprouvant } \\
\text { actuellement } \\
\text { l'enjeu }\end{array}$ & $\begin{array}{l}\text { Nombre de } \\
\text { patients } \\
\text { qui veulent } \\
\text { de l'aide }\end{array}$ & $\begin{array}{l}\text { Incertains } \\
\text { de vouloir } \\
\text { obtenir de } \\
\text { l'aide }\end{array}$ & $\begin{array}{l}\text { Parmi ceux qui } \\
\text { éprouvent l'enjeu, } \\
\text { proportion qui } \\
\text { veulent de l'aide } \\
\text { ou sont incertains } \\
\text { d'en vouloir } \mathbf{n} / \% \text { * }\end{array}$ \\
\hline Changements aux routines et au mode de vie & 25 & 7 & 0 & $7 / 28 \%$ \\
\hline Devoir attendre longtemps pour les rendez-vous cliniques & 13 & 8 & 1 & $9 / 69,2 \%$ \\
\hline Inquiétudes relatives à votre situation financière & 13 & 7 & 0 & $7 / 53,8 \%$ \\
\hline $\begin{array}{l}\text { Inquiétudes relatives aux déplacements à destination et en provenance } \\
\text { de l'hôpital }\end{array}$ & 12 & 8 & 1 & $9 / 75 \%$ \\
\hline Plus de choix quant à l'établissement où vous êtes soigné(e) & 9 & 2 & 1 & $3 / 33,3 \%$ \\
\hline Avoir des choix quant aux rendez-vous pour les tests et les traitements & 6 & 2 & 1 & $3 / 50 \%$ \\
\hline Plus de choix quant au cancérologue que vous consultez & 4 & 3 & 1 & $4 / 100 \%$ \\
\hline
\end{tabular}




\begin{tabular}{|c|c|c|c|c|}
\hline Enjeu & $\begin{array}{l}\text { Nombre de } \\
\text { patients } \\
\text { éprouvant } \\
\text { actuellement } \\
\text { l'enjeu }\end{array}$ & $\begin{array}{l}\text { Nombre de } \\
\text { patients qui } \\
\text { veulent de } \\
\text { l'aide }\end{array}$ & $\begin{array}{l}\text { Incertains } \\
\text { de vouloir } \\
\text { obtenir de } \\
\text { l'aide }\end{array}$ & $\begin{array}{l}\text { Parmi ceux qui } \\
\text { éprouvent l'enjeu, } \\
\text { proportion qui } \\
\text { veulent de l'aide ou } \\
\text { sont incertains d'en } \\
\text { vouloir n/\%* }\end{array}$ \\
\hline Manque d'énergie (PH) & 53 & 24 & 4 & $28 / 52,8 \%$ \\
\hline Douleur (PH) & 52 & 31 & 2 & $33 / 63,5 \%$ \\
\hline S’inquiéter du souci que se font les personnes qui vous sont chères (SO) & 51 & 12 & 3 & $15 / 29,4 \%$ \\
\hline Ne pas être capable de faire ce que vous pouviez faire auparavant (PH) & 49 & 20 & 3 & $23 / 46,9 \%$ \\
\hline Ne pas être capable de faire les travaux ménagers $(\mathrm{PH})$ & 43 & 17 & 2 & $19 / 44,2 \%$ \\
\hline Craintes relatives à la déficience ou à la détérioration physique (PS) & 42 & 17 & 4 & $21 / 50 \%$ \\
\hline Se sentir abattu(e) ou déprimé(e) (E) & 40 & 10 & 2 & $12 / 30 \%$ \\
\hline Ne pas se sentir bien (PH) & 38 & 13 & 4 & $17 / 44,7 \%$ \\
\hline Perte d'appétit (PH) & 38 & 15 & 3 & $18 / 47,4 \%$ \\
\hline Crainte que le cancer ne se propage (PS) & 35 & 15 & 3 & $18 / 51,4 \%$ \\
\hline Craintes relatives à la douleur (PS) & 35 & 19 & 3 & $22 / 62,9 \%$ \\
\hline
\end{tabular}

...suite de la page 96

voulaient nécessairement qu'on les aide à son propos. Même en ce qui a trait aux 11 items les plus fréquemment éprouvés, seulement 29,4 à 63,5\% des patients éprouvant actuellement un enjeu voulaient recevoir de l'aide à son sujet. Par contre, même si relativement peu de patients éprouvaient un enjeu particulier, une forte proportion des patients ayant ce besoin voulaient qu'on les aide à son propos. Par exemple, quoique les besoins d'information ne figurent pas parmi les 11 enjeux principaux, de 50 à $85,7 \%$ des patients qui éprouvaient des besoins dans ce domaine voulaient recevoir de l'aide.

Des résultats similaires relativement au désir d'être aidés des patients ont été obtenus auprès d'autres clientèles parvenues à divers stades du cancer et traitées dans cette clinique de cancérologie (Fitch \& Steele, 2010a; Fitch \& Steele, 2010b; Steele et al., 2004; Steele \& Fitch, 2008) et ont amené les membres du personnel à se demander si les patients savent réellement ce dont ils ont besoin. Cependant, une étude qualitative auprès de 34 patients du centre a révélé qu'il existe de multiples raisons pour lesquelles les patients déclaraient ne pas vouloir d'aide de la part du personnel à un moment particulier. Ces raisons incluaient notamment les suivantes: ils croyaient que le personnel était trop occupé, que le personnel ne serait pas intéressé par un certain type de problème, que le problème finirait par s'en aller de lui-même, ou bien qu'il n'y avait rien qui puisse être fait pour le type de problème éprouvé par la personne (Steele \& Fitch, 2008). Les participants à l'étude de Steele \& Fitch ont également dégagé plusieurs ressources externes ou communautaires qu'ils avaient utilisées plutôt que celles du centre de cancérologie, comme des membres de la famille, des amis et des professionnels de leur collectivité.

\section{Implications}

\section{Implications pour la pratique: symptômes}

Comme dans les études antérieures (p. ex. Bradley et al., 2005), le manque d'énergie constituait un problème de taille pour un grand nombre de patients atteints d'un cancer de stade avancé.
Une fatigue accablante nuit à la capacité de la personne à exécuter les activités quotidiennes normales (Fitch, Mings \& Lee, 2008) et les pertes associées peuvent avoir un effet dévastateur sur le bienêtre de la personne (Kennett \& Payne, 2005). Par contre, on dispose de résultats de recherche sur la façon de régler le problème de la fatigue. Les infirmières en oncologie doivent fournir aux patients des suggestions quant à la gestion de ce manque d'énergie, lesquelles pourraient se traduire par une amélioration de la qualité de vie chez ces patients (Fitch, Mings \& Lee, 2008).

La douleur est un autre enjeu important qui a été cerné dans des études antérieures (Bradley et al., 2005; Bruera et al., 2001). Étant donné la nature de la CIRRP, il n'est peut-être pas étonnant que tant de patients rapportent éprouver de la douleur. Toutefois, la portée de ce problème fait fortement valoir l'importance d'une maîtrise de la douleur plus efficace. Bien qu'il puisse être difficile de faire le suivi et l'évaluation de l'efficacité des soins de soutien auprès de ce groupe de patients, puisque beaucoup d'entre eux reçoivent leur traitement en une seule fois au centre de cancérologie, il importe d'instituer un suivi pour s'assurer qu'on a dispensé des soins optimaux. Le personnel des centres de cancérologie pourrait envisager d'utiliser d'un service de suivi téléphonique pour les patients en phase palliative afin d'évaluer l'atténuation des symptômes, plutôt que de s'attendre à ce que les patients reviennent à la clinique à des fins d'évaluation (Chow et al., 2001).

Les symptômes de dépression et d'anxiété constituaient également des problèmes courants chez les patients de la présente étude et pour lesquels ces derniers voulaient recevoir de l'aide. Les données probantes existantes montrent que ces symptômes peuvent avoir un effet adverse sur la qualité de vie des patients (Bruera et al., 2001; Bradley et al., 2005). Ces symptômes doivent eux aussi être évalués et documentés afin d'améliorer les références à des fins de gestion appropriée (Bradley et al., 2005). Bruera et ses collaborateurs (2001) ont démontré que les patients de leur clinique $(\mathrm{N}=166)$ obtenaient des améliorations psychosociales 
statistiquement significatives lorsqu'ils recevaient de l'aide de la part d'une équipe multiprofessionnelle. Il se peut que les centres de cancérologie doivent élargir leurs ressources professionnelles ou réévaluer les façons dont ils dispensent actuellement les soins afin de veiller à ce que les patients reçoivent l'aide la plus appropriée.

\section{Implications pour la pratique: inquiétudes psychosociales}

Les enjeux psychosociaux (c.-à-d. les items des domaines des besoins psychologiques, sociaux et affectifs) figuraient parmi les enjeux les plus fréquemment rapportés par les patients comme étant éprouvés au moment de la tenue de l'enquête. Ceux-ci ont également été cernés dans des écrits antérieurs (p. ex. Bradley et al., 2005; Bruera et al., 2001; Kennett \& Payne, 2005; Solano \& Higginson, 2006). On a de plus en plus d'indications que les interventions psychosociales pourraient rehausser le bien-être et améliorer la survie des patients atteints de cancer (Chow, Tsao \& Harth, 2004) et le bien-être des patients en phase palliative (Kennett \& Payne, 2005). Par exemple, Voogt et ses collègues (2005) suggéraient que les troubles de l'humeur chez les patients ayant un cancer avancé $(n=105)$ pouvaient être reliés à l'absence d'émotions positives plutôt qu'à la présence d'un haut niveau d'émotions négatives. Le manque d'émotions positives était lié à l'incapacité à s'impliquer dans des activités empreintes de sens et à la réduction du fonctionnement cognitif et du fonctionnement social et aussi à un surplus de fatigue. Si les professionnels parviennent à déterminer quelles activités sont porteuses de sens pour les patients et les aident à les poursuivre le plus longtemps possible ou suggèrent des activités de rechange, le cas échéant, il se peut que les patients éprouvent moins de détresse psychologique. En outre, on reconnaît de plus en plus qu'il est essentiel de mieux comprendre les groupes de symptômes et la manière dont nos interventions peuvent avoir une incidence sur plusieurs symptômes à la fois (Fitch \& Bultz, 2009).

\section{Implications pour la pratique:}

\section{fournir l'aide quand elle est souhaitée}

Il convient d'élaborer une méthode d'évaluation efficace et efficiente des besoins en matière de soins de soutien de tous les patients ayant un cancer avancé et ce, de façon systématique. Les problèmes les plus fréquemment dégagés dans le cadre de la présente étude pourraient servir à développer un outil de dépistage ou de triage qui repérerait les enjeux actuellement éprouvés, la mesure dans laquelle ceux-ci sont éprouvés et si le patient souhaite recevoir ou non de l'aide à leur propos. Cette étape de triage préliminaire pourrait être à la base d'une conversation avec le patient qui pourrait aboutir à une évaluation en profondeur des problèmes auxquels la personne se trouve confrontée. L'exploration des attentes des patients en matière d'aide permettrait aux infirmières en oncologie et aux patients de discuter des prochaines étapes éventuelles grâce auxquelles les patients recevraient le type d'aide qu'ils désirent.

L'outil utilisé dans la présente étude fournissait le type requis d'évaluation globale des besoins pour l'éventail complet des soins de soutien. La mise au jour de problèmes donne aux professionnels l'occasion de fournir de l'aide. En revanche, les professionnels ne doivent pas présumer qu'un problème particulier ne constitue pas un problème pour un patient donné si ce dernier ne l'a pas mentionné. Ils ne doivent pas non plus présumer qu'un problème particulier ne vaut pas vraiment la peine d'être abordé parce que fort peu de personnes l'éprouvent. Il importe de demander aux patients s'ils éprouvent un problème et s'ils désirent recevoir de l'aide à son sujet. Bien qu'un nombre important de patients souhaitent qu'on les aide à régler leurs problèmes, il y en a d'autres qui ne veulent pas se prévaloir d'aide, et il convient d'honorer les souhaits des patients. Une discussion avec les patients pourrait établir une compréhension mutuelle entre le patient et le prestataire de soins sur ce qui pourrait lui être d'une plus grande aide et ce, depuis la perspective du patient. Les professionnels de la santé se doivent de reconnaître l'existence de problèmes psychologiques, tels que les craintes relatives à la déficience ou à la détérioration physique, ainsi que celle de problèmes physiques et d'entretenir un dialogue et une communication ouverts avec les patients afin de pouvoir aiguiller ces derniers vers les ressources où ils trouveront l'aide nécessaire.

\section{Implications pour les ressources et les politiques}

L'importante proportion des patients indiquant qu'ils aimeraient recevoir de l'aide afin de régler des problèmes particuliers donne à penser que de nombreux patients recherchaient une assistance dépassant celle qui leur est actuellement fournie. Il est manifeste que même dans les bons centres de cancérologie, les patients avaient encore des besoins non satisfaits. Cette observation a des conséquences pour la planification des programmes, l'affectation des ressources et la recherche, à la fois dans les cliniques d'intervention rapide et dans d'autres contextes des centres de cancérologie où les patients atteints de cancers avancés reçoivent leurs soins.

Les centres de cancérologie ne disposent pas des ressources leur permettant de fournir tous les services à tous leurs patients; pourtant, ils ont des responsabilités envers les patients ayant un cancer avancé. Les normes prônant des soins de cancérologie de qualité soulignent que le traitement contre le cancer doit être optimal dans toute la gamme des soins. Dans un même temps, il est possible que les centres de cancérologie jugent qu'ils ne peuvent mettre qu'un ensemble limité de ressources au service de la clientèle des patients atteints d'un cancer avancé qui ne le fréquentent que pour un ou deux traitements. Il en ressort que la planification et l'affectation des ressources doivent être traitées de manière différentielle entre les patients du centre à court terme, d'une part, et à long terme, d'autre part. En ce qui concerne la CIRRP, le personnel pourrait dégager certains problèmes chez les patients ayant fait l'objet de l'évaluation et informer de ces problèmes les prestataires chargés de leurs soins primaires en dehors du centre. Lorsque les soins sont dispensés en collaboration avec les médecins de la collectivité qui continuent d'assurer la gestion globale des soins palliatifs, le personnel du centre de cancérologie pourrait remettre aux patients une lettre au sujet de leur traitement et inclure des renseignements sur les problèmes qui doivent être abordés. Quoique cette intervention puisse être interprétée comme étant une directive adressée aux médecins de premier recours, elle permet de garder les besoins des patients et leur désir d'être aidés à leur propos au centre des préoccupations des professionnels de la santé dans tout le continuum des soins axés sur le patient.

Il est également primordial de discuter avec les patients de toutes les ressources appropriées et interventions disponibles. Idéalement parlant, cette discussion devrait être réalisée par l'infirmière en oncologie dans le cadre des soins standard. Dans certaines régions, des centres pourraient déployer des infirmières en pratique avancée afin de dispenser les soins dans les situations les plus complexes. Les résultats de la présente étude démontrent que les problèmes existent bel et bien, et les normes de soins indiquent clairement que ceux-ci doivent être abordés (Agrément Canada, 2010). En fin de compte, ces données peuvent servir à justifier ou appuyer le financement de programmes en soins de soutien, la détermination des interventions et le développement de protocoles basés sur des résultats de recherche.

\section{Implications pour la recherche}

Les résultats novateurs dégagés dans le cadre de cette étude justifient la tenue de nouveaux projets de recherche. Par exemple, on pourra approfondir les raisons pour lesquelles les patients atteints d'un cancer avancé déclarent qu'ils ne souhaitent pas recevoir d'aide dans certains domaines de besoin, les ressources existant actuellement à l'extérieur du centre de cancérologie qu'ils utilisent pour essayer de satisfaire leurs besoins et quelles ressources (au centre de cancérologie ou à l'extérieur) pourraient leur être utiles. Une analyse documentaire approfondie pourrait être réalisé afin 
d'explorer les trois plus grands enjeux cernés par cette étude (notamment une approche dyadique mettant en jeu les soignants naturels qui s'occupent de ces patients chez eux), et de développer des interventions locales pour les problèmes qui sont éprouvés au moyen d'un essai clinique aléatoire afin de comparer les soins standard à l'intervention basée sur les besoins particuliers du patient et sur son désir d'être aidé. Les patients et les soignants naturels pourraient être suivis dans le cadre d'une étude longitudinale afin de dégager les interventions qui se sont avérées efficaces et pour qui, et de cerner les mécanismes ou processus médiateurs qui permettent d'expliquer pourquoi la ou les intervention(s) ont donné des résultats. Finalement, les améliorations psychosociales pourraient être évaluées dans les cliniques offrant de l'aide dispensée par une équipe multiprofessionnelle.

\section{Conclusion}

Les patients ayant un cancer avancé et traités en ambulatoire dans une clinique de radiothérapie palliative faisaient état de

\section{RÉFÉRENCES}

Agrément Canada (2010). Les normes: Services de traitement du cancer et oncologie. Ottawa: Auteur.

Andersson. L., \& Sousa, P. (1998). An innovative way of caring: Palliative radiation therapy rapid response clinic. Canadian Oncology Nursing Journal, 8(2), 146-147.

Ashbury, F.D., Findley, H., Reynolds, B., \& McKerracher, K. (1998). A Canadian survey of cancer patients' experiences: Are their needs being met? Journal of Pain and Symptom Management, 16(5), 298-306.

Body, J. (1992). Metastatic bone cancer: Clinical and therapeutic aspects. Bone, 13, 1350-1354.

Bonevski, B., Sanson-Fisher, R., Girgis, A., Burton, L., Cook, P., Boyes, A., \& the Supportive Care Review Group. (2000). Evaluation of an instrument to assess the needs of patients with cancer. Cancer, 88(1), 217-225.

Bradley, N., Davis, L., \& Chow, E. (2005). Symptom distress in patients attending an outpatient palliative radiotherapy clinic. Journal of Pain and Symptom Management, 30(2), 123-131.

Bruera, E., Michaud, M., Vigano, A., Neumann, C., Watanabe, S., \& Hanson, J. (2001). Multidisciplinary symptom control clinic in a cancer centre: A retrospective study. Support Care Cancer, 9, 162-168.

Charles, K., Sellick, S.M., Montesanto, B., \& Mohide, E.A. (1996). Priorities for cancer survivors regarding psychosocial needs. Journal of Psychosocial Oncology, 14(2), 57-72.

Chow, E., Tsao, M., \& Harth, T. (2004). Does psychosocial intervention improve survival in cancer? A meta-analysis. Palliative Medicine, $18,25-31$.

Chow, E., Wong, R., Connolly, R., Hruby, G., Franssen, E., Fung, K., et al. (2001). Prospective assessment of symptom palliation for patients attending a rapid response radiotherapy program: Feasibility of telephone follow-up. Journal of Pain and Symptom Management, 22(2), 649-656.

Ciezki, J., Komurcu, S., \& Macklis, R. (2000). Palliative radiotherapy. Seminars in Oncology, 27(1), 90-93.

Edmonds, P., Karlsen, S., Khan, S., \& Addington-Hall, J. (2001). A comparison of the palliative care needs of patients dying from chronic respiratory diseases and lung cancer. Palliative Medicine, 15(4), 287-295.

Fitch, M.I., Mings, D., \& Lee, A. (2008). Exploration des expériences des patients en matière de fatigue liée au cancer et de leurs stratégies personnelles pour y faire face. Revue canadienne de soins infirmiers en oncologie, 18(3), 132-140.

Fitch, M.I., \& Bultz, B. (2009). Symptom patterns in patients with advanced cancer. Oncology Exchange, 8(4),11-13. symptômes et de besoins psychosociaux similaires à ceux d'autres patients atteints de cancers avancés. Toutefois, l'identification de ceux qui éprouvaient actuellement un enjeu particulier ne signifiait pas nécessairement qu'ils voulaient recevoir de l'aide à son sujet. Fait paradoxal, pour certains items où relativement peu de patients éprouvaient un problème, un plus fort pourcentage d'entre eux voulaient de l'aide à son propos. Les infirmières en oncologie doivent évaluer les besoins particuliers des patients dans de multiples domaines (p. ex. physique, psychosocial, spirituel, informationnel, pratique), mais elles ne doivent pas oublier non plus que certains patients ne veulent pas qu'on les aide. À tout moment donné, ce qui importe est que les infirmières et les patients entament un dialogue en vue de déterminer ce qui constitue une préoccupation et quelle aide peut être offerte pour elle et est valable aux yeux du patient. La fourniture d'une gamme élargie d'aide pourra être utile aux patients, mais les cliniques doivent planifier et développer leurs interventions de manière à répondre aux besoins particuliers de leur clientèle tout en maximalisant leurs ressources.

Fitch, M.I., Porter, H.B., \& Page, B.D. (2008). Supportive Care Framework: A Foundation for Person-Centered Care. Pembroke, ON: Pappin Communications.

Fitch, M.I., \& Steele, R. (2010a). Dégager les besoins en soins de soutien des femmes atteintes d'un cancer ovarien. Revue canadienne de soins infirmiers en oncologie, 20(2), 66-74.

Fitch, M.I., \& Steele, R. (2010b). Besoins en soins de soutien des personnes atteintes de cancer du poumon. Revue canadienne de soins infirmiers en oncologie. Offert en ligne dans le site Web de l'ACIO.

Hutton, J., Martin, L., Field, C.J., Wismer, W.V., Bruera, E.D., Watanabe, S.M., et al. (2006). Dietary patterns in patients with advanced cancer: Implications for anorexia-cachexia therapy. American Journal of Clinical Nutrition, 84(5), 1163-1170.

Kennett, C., \& Payne, M. (2005). Understanding why palliative care patients 'like day care' and 'getting out'. Journal of Palliative Care, 21(4), 292-298.

Kirkbride, P. (1995). The role of radiation therapy in palliative care. Journal of Palliative Care, 11(1), 19-26.

Sarna, L., Evangelista, L., Tashkin, D., Padilla, G., Holmes, C., Brecht, M., et al. (2004). Impact of respiratory symptoms and pulmonary function on quality of life of long-term survivors of non-small cell lung cancer. Chest, 125(2), 439-445.

Sarna, L., Lindsey, A.M., Dean, H., Brecht, M.L., \& McCorkle, R. (1994). Weight change and lung cancer: Relationships with symptom distress, functional status and smoking. Research in Nursing \& Health, 17(5), 371-379.

Solano, J., \& Higginson, I. (2006). A comparison of symptom prevalence in far advanced cancer, AIDS, heart disease, chronic obstructive pulmonary disease and renal disease. Journal of Pain and Symptom Management, 31(1), 58-68.

Steele, R., Fitch, M.I., Alderson, D., Barrow, K., Hurst, N., \& Doyle, C. (2004). Measuring supportive care needs of women with gynecological cancer. Supportive Care in Cancer, 12(6), 411.

Steele, R., \& Fitch, M.I. (2008). Why patients with lung cancer do not want help with some needs. Supportive Care in Cancer, 16(3), 251-259.

Voogt, E., van der Heide, A., van Leeuwen, A.F., Visser, A.P., Cleiren, M.P., Passchier, J., et al (2005). Positive and negative affect after diagnosis of advanced cancer. Psychooncology, 14(4), 262-273.

Whelan, T.J., Mohide, E.S., Willan, A.R., Arnold, A., Tew, A., Sellick, S., et al. (1997). The supportive care needs of newly diagnosed cancer patients attending a regional cancer centre. Cancer, 80(8), 1518-1524. 